\title{
Role of Physisorption States in Molecular Scattering: A Semilocal Density-Functional Theory Study on $\mathrm{O}_{2} / \mathrm{Ag}(111)$
}

\author{
I. Goikoetxea, ${ }^{1, *}$ J. Meyer, ${ }^{2, \dagger}$ J. I. Juaristi, ${ }^{1,3,4}$ M. Alducin, ${ }^{1,4}$ and K. Reuter ${ }^{2}$ \\ ${ }^{1}$ Centro de Física de Materiales CFM/MPC (CSIC-UPV/EHU), Paseo Manuel de Lardizabal 5, E-20018 San Sebastián, Spain \\ ${ }^{2}$ Theoretical Chemistry and Catalysis Research Center, Technische Universität München, \\ Lichtenbergstrasse 4, D-85747 Garching, Germany \\ ${ }^{3}$ Departamento de Física de Materiales, Facultad de Químicas, UPV/EHU, Apartado 1072, E-20080 San Sebastián, Spain \\ ${ }^{4}$ Donostia International Physics Center DIPC, Paseo Manuel de Lardizabal 4, E-20018 San Sebastián, Spain
}

(Received 23 January 2014; published 16 April 2014)

\begin{abstract}
We simulate the scattering of $\mathrm{O}_{2}$ from $\mathrm{Ag}(111)$ with classical dynamics simulations performed on a six-dimensional potential energy surface calculated within semilocal density-functional theory. The enigmatic experimental trends that originally required the conjecture of two types of repulsive walls, arising from a physisorption and chemisorption part of the interaction potential, are fully reproduced. Given the inadequate description of the physisorption properties in semilocal density-functional theory, our work casts severe doubts on the prevalent notion to use molecular scattering data as indirect evidence for the existence of such states.
\end{abstract}

DOI: 10.1103/PhysRevLett.112.156101

PACS numbers: 68.49.Df, 34.35.+a, 82.20.Kh

Molecular scattering experiments at single-crystal surfaces have a long tradition in the analysis of moleculesurface interaction and energy loss mechanisms, which are both key aspects in the quest to understand gas-surface dynamics [1-3]. A prevalent notion in the analysis of this kind of scattering data is that it is critically influenced by the potential energy surface (PES) topology at large distances from the surface. As physisorption states are located a such large distances, there is a general perception that scattering experiments are rather sensitive to them, or vice versa, that scattering experiments provide detailed information on such kind of states.

In this understanding, it is puzzling to realize that several recent first-principles studies modeling the scattering dynamics at metal surfaces have reached quite satisfactory, if not quantitative, descriptions of corresponding experimental data [4-8]. In these studies the high-dimensional PES underlying the dynamical simulations is generally obtained from density-functional theory (DFT) with semilocal exchange-correlation (xc) functionals. By construction, such short-ranged functionals cannot provide an adequate description of van der Waals interactions [9-12], and the corresponding PES will not feature any weakly bound physisorption states far away from the surface.

In order to further elucidate this point, we focus here on $\mathrm{O}_{2} / \mathrm{Ag}(111)$, which is not only a prototypical system allegedly featuring a long-discussed (but still rather elusive) physisorption state [13-17], but also a system where detailed scattering data have been centrally interpreted in terms of a repulsive wall separating this physisorption from a stronger bound chemisorption state. In contrast, the PES from semilocal DFT that has already been used to successfully describe the molecular sticking data at this surface
[18] does not feature a physisorption well, and as such this system represents an intriguing test case to pinpoint the role of physisorption states for molecular scattering.

The scattering of $\mathrm{O}_{2}$ from $\mathrm{Ag}(111)$ is simulated following the divide-and-conquer approach we already used in the study of the $\mathrm{O}_{2}$ dissociative adsorption on this surface [18]. This approach requires as a first step the calculation of an accurate multidimensional PES, which is subsequently used for evaluating the gas-surface dynamics. Here, the ground-state PES along the six $\mathrm{O}_{2}$ molecular degrees of freedom at a rigid $\operatorname{Ag}(111)$ surface is first extensively mapped with DFT calculations performed with the semilocal xc functional due to Perdew, Burke, and Ernzerhof [19]. Infinite molecule-surface separation defines the zeroenergy reference. A neural networks-based technique $[20,21]$ that properly exploits the symmetry of the studied system $[18,22]$ then yields a continuous six-dimensional (6D) PES. On this PES representation, millions of classical molecular dynamics trajectories are finally integrated at low computational cost.

The scattering of $\mathrm{O}_{2}$ from $\mathrm{Ag}(111)$ was extensively studied by Raukema et al. using a supersonic molecular beam apparatus that permitted incidence energies $0.4<$ $E_{i}<1.8 \mathrm{eV}$ at defined incidence angles $\left(\Theta_{i}, \Phi_{i}\right)$ [23]. Compared with their results for $\mathrm{N}_{2}$ scattering and largely independent of the azimuthal angle $\Phi_{i}$, the in-plane angular distributions $\Theta_{f}$ of the scattered $\mathrm{O}_{2}$ molecules exhibit a peculiar variation with $E_{i}$ and $\Theta_{i}$ : At $\Theta_{i}=40^{\circ}$, cf. Fig. 1 for a definition of the scattering geometry, the distribution is narrowly peaked around specular reflection for low incidence energies, $E_{i} \simeq 0.4 \mathrm{eV}$. At $E_{i}$ increasing above $1.0 \mathrm{eV}$, the distribution instead rapidly broadens towards the surface normal, concomitant with a decreasing intensity 
at specular reflection. This $E_{i}$ dependence is intriguingly smoothly reversed, when the polar incidence angle varies from $40^{\circ}$ to $70^{\circ}$. At the largest angle $\left(\Theta_{i}=70^{\circ}\right)$, symmetric and narrow angular distributions around specular are obtained for all incidence energies. However, in contrast to the $\Theta_{i}=40^{\circ}$ case, it is at the largest incident energy, when the distribution is the narrowest and the height of the distribution peak the largest.

In Ref. [23] these results were rationalized in terms of scattering from a dual repulsive wall arising from the physisorption and chemisorption part of the interaction potential. In this picture, low normal-energy molecules are scattered at large distances from the surface in the repulsive walls connected with the shallow physisorption state, where the PES is still rather uniform in its lateral degrees of freedom. In contrast, high normal-energy molecules overcome these first barriers and are reflected closer to the surface on the repulsive walls related with the chemisorption state where the PES is more corrugated. The broadening of the angular distributions for the more normal incidence $\Theta_{i}=40^{\circ}-60^{\circ}$ is thus explained by an increase in lateral PES corrugation experienced by the more energetic $\mathrm{O}_{2}$ molecules. In contrast, at the most grazing $\Theta_{i}=70^{\circ}$ all molecules would already be reflected by the walls of the physisorption state. In this case, the varying width of the angular distributions was associated with thermal broadening, i.e., the influence of the surface atom vibrations.

Quite common for the interpretation of scattering data, this picture assumes the existence of two separate adsorption wells, a shallow physisorption state and a deeper chemisorption state. This is at variance with the previous analysis of our calculated 6D DFT PES [18], whose high interpolation quality is guaranteed by the small root mean square error (RMSE) $(<26 \mathrm{meV})$ in dynamically important regions. There is indeed a molecular chemisorption well and a steep repulsive wall with energies of more than $1 \mathrm{eV}$ required to bring the molecule closer than $2 \AA$ to the surface. The chemisorption well is only $-40 \mathrm{meV}$ deep (though $-70 \mathrm{meV}$ if surface relaxation is allowed), which already casts severe doubts on its actual role for the scattering data. Moreover, in the DFT PES, calculated using a semilocal $\mathrm{xc}$ functional, physisorption wells are entirely absent. In this respect, multidimensional dynamics simulations on this PES are ideally suited to elucidate which PES topological features really rule the peculiar $\mathrm{O}_{2}$ scattering off $\operatorname{Ag}(111)$.

With this objective we perform classical trajectory calculations for defined $E_{i}$ (neglecting the initial zero-point energy) and $\Theta_{i}$. For each $\left(\Theta_{i}, E_{i}\right)$ condition, $10^{6}$ trajectories sample over all possible initial $\mathrm{O}_{2}$ angular orientations and lateral positions over the surface unit cell. Consistent with experiment, we do not observe any specific dependence on the azimuthal incidence angle. At an unspecified crystal orientation in the measurements, the trajectory ensemble correspondingly also averages over this degree of freedom.
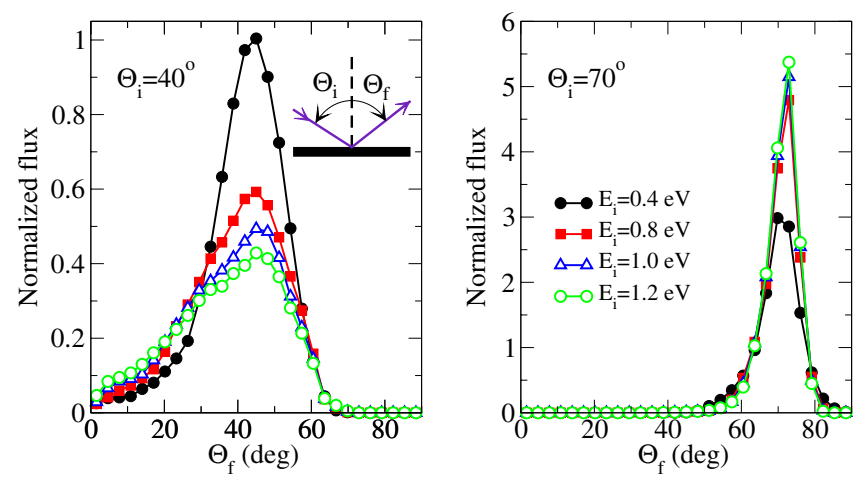

FIG. 1 (color online). Calculated angular distributions for inplane $\mathrm{O}_{2}$ scattering off $\mathrm{Ag}(111)$ for incidence angles $\Theta_{i}=40^{\circ}$ (left) and $\Theta_{i}=70^{\circ}$ (right) and various $E_{i}$. All fluxes are normalized to the peak height obtained for $\Theta_{i}=40^{\circ}$ and $E_{i}=0.4 \mathrm{eV}$.

The in-plane scattering angle distributions $\Theta_{f}$ obtained from these simulations are shown in Fig. 1 for the two extreme incidence angles used in experiment, namely, $\Theta_{i}=40^{\circ}$ and $\Theta_{i}=70^{\circ}$. Following Ref. [23], all curves are normalized to the maximum value of the distribution we obtain for $\Theta_{i}=40^{\circ}$ and $E_{i}=0.4 \mathrm{eV}$. For each incidence condition $\left(\Theta_{i}, E_{i}\right)$, the in-plane distribution is calculated by selecting the molecules that are reflected within a fixed solid angle. This means that for each $\Theta_{f}$ the acceptance azimuthal angle is given by $\left|\Phi_{f}-\Phi_{i}\right|<$ $\Delta \Phi^{0} / \sin \Theta_{f}$, where $\Delta \Phi^{0}$ is the acceptance azimuthal angle at $\Theta_{f}=90^{\circ}$. The value $\Delta \Phi^{0}=5^{\circ}$ that has been used in calculating the distributions assures reliable statistics even at extreme observation conditions where reflection events are less probable.

In full agreement with experiment, we observe that the decrease in the distribution maximum is accompanied by an increase of the curve width. In fact, despite the absence of physisorption states in the DFT PES, our simulations reproduce rather well all general experimental trends described above. Whereas for $\Theta_{i}=40^{\circ}$ the distributions broaden towards the surface normal and the height of the specular peak monotonically decreases as $E_{i}$ increases, for $\Theta_{i}=70^{\circ}$ the broadening is rather symmetric and the peak behavior is just the opposite. Note that the peak decrease is not only a consequence of the broadening in the in-plane polar distribution, but is also related to an increase in outof-plane scattering events. The change in the peak height is explicitly compared to the experimental data in Fig. 2. In both cases [Figs. 2(a) and 2(b)] the decrease of the peak height with increasing $E_{i}$ is gradually turned into an increase as the incidence angle is varied from $40^{\circ}$ to $70^{\circ}$.

The lower panels of Fig. 3 provide first insight into the complex and variable energy landscape that the molecules can encounter while approaching the $\operatorname{Ag}(111)$ surface. In the conceptual one-dimensional picture underlying the experimental data analysis [23], the potential energy 

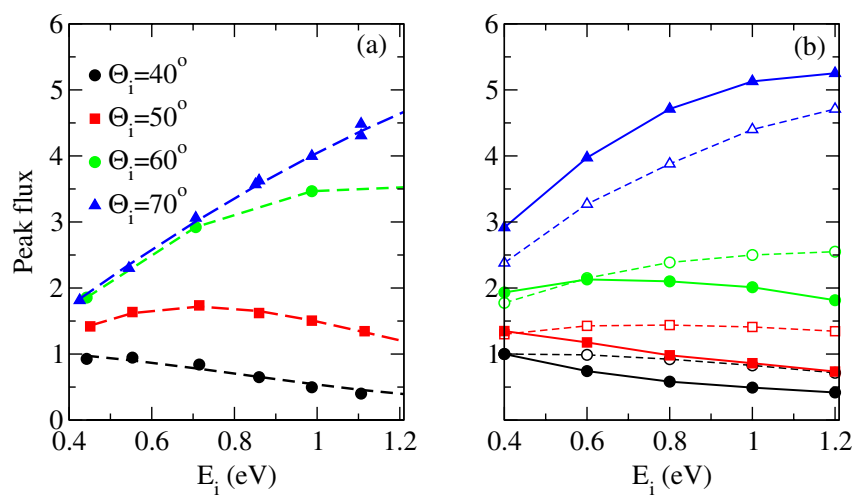

FIG. 2 (color online). Peak height of the distributions of Fig. 1 as a function of incidence energy $E_{i}$. The experimental data reproduced from Ref. [23] (left) can be directly compared with the results of our adiabatic (full lines) and GLO (dashed lines) simulations (right). Lines through the data points are guides to the eye.

(PE) as a function of the distance $Z$ from the surface, $E=E(Z)$, exhibits two wells separated by a barrier and a repulsive wall at distances closer than the chemisorption well. Depending on the $\mathrm{O}_{2}$ orientation $(\theta, \phi)$ and lateral position $(X, Y)$ over the surface unit cell, the true 6D PES $E(X, Y, Z, d, \theta, \phi)$ as a function of $Z$ exhibits a range of values stretching over several eV, even if restricting the $\mathrm{O}_{2}$ internuclear distance $d$ to values between the one in gas
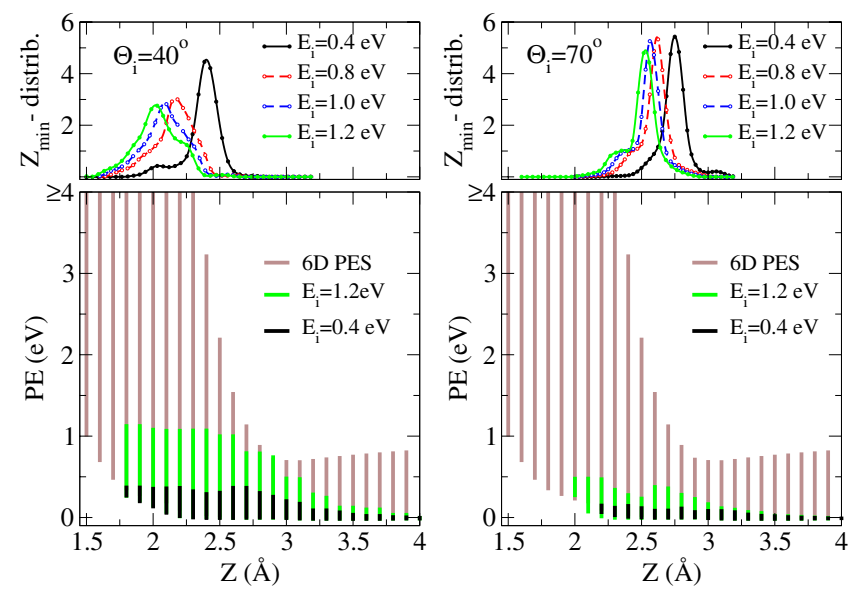

FIG. 3 (color online). Upper panels: Distributions of the molecule's closest approach distance $Z_{\min }$ to the surface for the different incidence conditions. The distribution curve for each $\Theta_{i}$ and $E_{i}$ is normalized to the corresponding total number of inplane scattered molecules. Lower panels: Range of PE values exhibited by the 6D PES $E(X, Y, Z, d, \theta, \phi)$ as a function of distance $Z$ to surface (maroon vertical lines) as compared to the $\mathrm{PE}$ range that is actually probed by the $\mathrm{O}_{2}$ molecules along their trajectories for $E_{i}=0.4 \mathrm{eV}$ (black vertical lines) and $E_{i}=$ $1.2 \mathrm{eV}$ (green vertical lines); see text. Left panels correspond to $\Theta_{i}=40^{\circ}$, right panels to $\Theta_{i}=70^{\circ}$. Incidence angles in between (not shown) exhibit a smooth transition between these two limits. phase $(1.24 \AA)$ and that at the chemisorption state $(1.28 \AA)$. At this complexity, it is enlightening to analyze which of these PE values are actually encountered by the incoming $\mathrm{O}_{2}$ molecules for the varying incidence conditions. As shown in Fig. 3, the incidence energy can effectively be used to reach regions of PEs up to almost $E_{i}$ for $\Theta_{i}=40^{\circ}$, whereas for the more grazing $\Theta_{i}=70^{\circ}$, such regions are inaccessible. Most importantly, the PE regions visited in the latter case always stay below the normal energy component of $E_{i}$, whereas for $\Theta_{i}=40^{\circ}$ this is only the case for the lowest $E_{i}=0.4 \mathrm{eV}$. This analysis thus supports the effective "dual-repulsive" wall picture of Raukema et al:: At $\Theta_{i}=70^{\circ}$ (and $\Theta_{i}=40^{\circ}, E_{i}=0.4 \mathrm{eV}$ ), the $\mathrm{O}_{2}$ molecules are dominantly scattered at energy barriers in the entrance channel, i.e., at distances far from the surface where at low lateral PES corrugation the dynamics is still governed by the normal energy [24-26]. At the other incidence conditions, corresponding to normal energies exceeding $0.25 \mathrm{eV}$, the molecules get closer to the surface and are scattered at more corrugated PES parts where the normalenergy condition is no longer obeyed.

This view of the scattering at two types of repulsive regions is further confirmed by the analysis of the distance of closest approach $Z_{\min }$ of the in-plane reflected molecules. The results plotted in the upper panels of Fig. 3 clearly demonstrate that there are two distinct regions where the molecules are preferentially reflected. At incidence conditions corresponding to low normal energies, the $Z_{\text {min }}$ distributions are characterized by a quite narrow peak at distances above $2.3 \AA$ and a small tail extending to closer distances from the surface. In these cases, the dominant fraction of the molecules is thus reflected above $2.3 \AA$ from the surface. In contrast, for incidence conditions corresponding to normal energies above $0.25 \mathrm{eV}$, the distributions are visibly shifted to smaller $Z_{\min }$ values and exhibit a very broad shape. Such broadening is clearly related to an increase in the corrugation of the PES close to the surface.

With this understanding, we can thus fully rationalize the inversion of the peak height variation with incidence energy for the two incidence angles. At $\Theta_{i}=40^{\circ}$ and $E_{i}>0.4 \mathrm{eV}$, the majority of the molecules are reflected below $2.3 \AA$, where the lateral PES corrugation is already important. The observed decrease in the height of the specular reflection peak at this incidence angle with increasing $E_{i}$ is thus a consequence of the larger PES corrugation probed by the molecules getting closer to the surface. In contrast, at $\Theta_{i}=$ $70^{\circ}$ the normal energy for all $E_{i}$ is so small that most of the molecules are reflected far from the surface without probing the corrugated part of the PES. Intriguingly, here our simulations at a rigid $\mathrm{Ag}(111)$ surface fully reproduce the decrease of the height of the specular peak with decreasing $E_{i}$ that was originally ascribed to thermal broadening. To further scrutinize that surface vibrations are indeed not key to this effect, we carried out additional simulations using the generalized Langevin oscillator 
Experiments

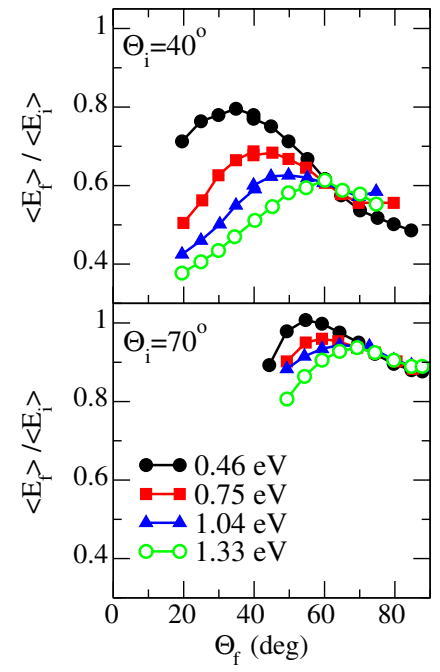

GLO

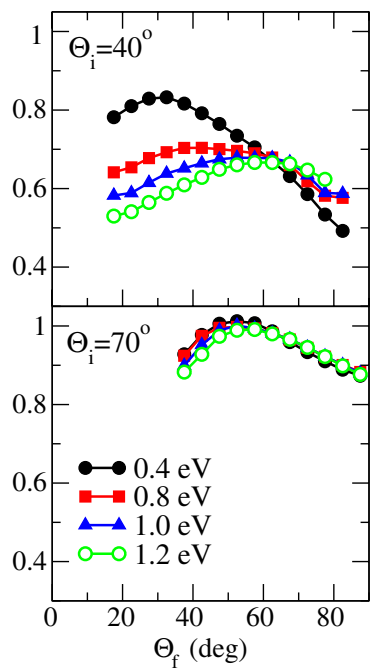

FIG. 4 (color online). Angularly resolved final-to-initial energy distributions of $\mathrm{O}_{2}$ scattered off $\mathrm{Ag}(111)$ under different incidence conditions $\left(\Theta_{i}, E_{i}\right)$. The experimental data by Raukema et al. [23] (left panels) are compared to the results from the GLO calculations (right panels); see text.

(GLO) model to mimic surface mobility [27,28]. The simulations are performed following the implementation of Ref. [29] (technical details on how the GLO is applied to the $\mathrm{Ag}(111)$ surface can be found in Ref. [30]). The GLO results, shown as dashed lines in Fig. 2(b), are indeed very similar to those obtained within the adiabatic frozensurface approximation. This confirms that the peak height decrease arises mostly from the small PES variations of about $20-30 \mathrm{meV}$ at distances $Z>3 \AA$, which become increasingly irrelevant as the normal incidence energy of the molecules increases. Note that for an incidence angle of $60^{\circ}$ there is a qualitative difference between our adiabatic calculations and the GLO results, which reproduce better the experimentally observed trend. This shows that, though it is not the dominant mechanism, some effect due to surface vibrations cannot be disregarded.

In addition to the scattering angular distributions, Raukema et al. measured the energy loss distribution of the scattered molecules [23]. These additional results were also interpreted as a further validation of the dual-repulsive wall picture. The experimental energy loss distributions are reproduced in Fig. 4. This figure shows the angularly resolved final-to-initial translational energy ratio distributions of $\mathrm{O}_{2}$ scattered from $\mathrm{Ag}(111)$ at different incidence conditions. Within this kind of representation, values of $\left\langle E_{f}\right\rangle /\left\langle E_{i}\right\rangle<1$ show that the molecule has lost translational energy after reflection (the larger the energy loss, the smaller the energy ratio). For $\Theta_{i}=70^{\circ}$, the energy loss distributions measured at different incidence energies reasonably follow the behavior expected for scattering from a flat PES, for which parallel momentum is conserved. This is precisely the behavior usually identified with physisorptionlike interactions. However, as $\Theta_{i}$ decreases, the final-to-initial energy curves are no longer subject to parallel momentum conservation. At the smallest incidence angle $\left(\Theta_{i}=40^{\circ}\right)$, the $\mathrm{O}_{2}$ scattering is instead characterized by high-energy losses. In fact, under large normal incidence energy conditions, the measured energy losses for outgoing angles $\Theta_{f} \leq 60^{\circ}$ can exceed those predicted within the binary-collision model, which is the manifestation of a highly corrugated PES. This result was interpreted as an indication of the molecules being reflected in a region close to the surface.

The final-to-initial energy distributions calculated with the GLO model are shown in the right-hand panels of Fig. 4. In all cases, the qualitative agreement between the results of our simulations and the experimental data is remarkable. In spite of its simplicity, the GLO model reproduces the shape and trends of the $\left\langle E_{f}\right\rangle /\left\langle E_{i}\right\rangle$ distributions, as well as the dependence on $E_{i}$ and $\Theta_{i}$. We state that this is a further validation of the quality of our PES and its capability to capture the main characteristics of the scattering dynamics.

In summary, we have studied the scattering properties of $\mathrm{O}_{2}$ molecules incident on the $\mathrm{Ag}(111)$ surface. Simulating the detailed scattering properties at various incidence angles and energies, we can fully reproduce the enigmatic trends that originally required the conjecture of two types of repulsive walls in the PES, arising from a physisorption and chemisorption part of the interaction potential. In fact, a detailed analysis of the simulated trajectories nicely confirms this concept of two repulsive walls. However, this is in the absence of any physisorption state in our DFT PES. The solution to this puzzle comes with the realization that scattering is not sensitive to the physisorption well itself, but instead to the repulsive wall "behind" a well. These walls typically emerge out of rehybridization phenomena (the molecular states need to orthogonalize to the electronic states of the surface), and semilocal DFT can describe this aspect rather reliably. Furthermore, it is only in an effective one-dimensional picture that a wall necessarily separates two distinct wells. In higher dimensions this is no longer true, and the existence of different repulsive regions in the PES must not necessarily imply the existence of a separate physisorption state. As such, our work casts severe doubts on the prevalent notion of using molecular scattering data as indirect evidence for the existence of such states. As with many other techniques, prominently, temperatureprogrammed desorption, such weakly bound states far away from the surface remain hard to grasp quantitatively and their real relevance for gas-surface dynamics remains uncertain.

The work of I. G. has been supported by the Spanish Research Council (CFM-CSIC, Grants No. JAEPre_08_0045 and No. 2010ESTCSIC-02167). Funding by the Deutsche Forschungsgemeinschaft is gratefully acknowledged. M. A. and J. I. J. acknowledge financial 
support by the Basque Departamento de Educación, Universidades e Investigación, the University of the Basque Country UPV/EHU (Grant No. IT-756-13), and the Spanish Ministerio de Ciencia e Innovación (Grant No. FIS2010-19609-C02-02).

*Also at Institut für Chemie, Humboldt Universität zu Berlin, Unter den Linden 6, D-10009 Berlin, Germany.

${ }^{\dagger}$ Also at Leiden Institute of Chemistry, Gorlaeus Laboratories, Leiden University, P.O. Box 9502, 2300 RA Leiden, Netherlands.

[1] J. Toennies, Appl. Phys. 3, 91 (1974).

[2] A. W. Kleyn, Chem. Soc. Rev. 32, 87 (2003).

[3] L. Vattuone, L. Savio, F. Pirani, D. Cappelletti, M. Okada, and M. Rocca, Prog. Surf. Sci. 85, 92 (2010).

[4] P. Nieto, E. Pijper, D. Barredo, G. Laurent, R. A. Olsen, E.-J. Baerends, G.-J. Kroes, and D. Farías, Science 312, 86 (2006).

[5] C. Díaz, J. K. Vincent, G. P. Krishnamohan, R. A. Olsen, G.-J. Kroes, K. Honkala, and J. K. Nørskov, J. Chem. Phys. 125, 114706 (2006).

[6] K. R. Geethalakshmi, J. I. Juaristi, R. Díez Muiño, and M. Alducin, Phys. Chem. Chem. Phys. 13, 4357 (2011).

[7] M. N. Batista, H. F. Busnengo, and A. E. Martínez, Phys. Chem. Chem. Phys. 13, 4614 (2011).

[8] F. Nattino, C. Díaz, B. Jackson, and G.-J. Kroes, Phys. Rev. Lett. 108, 236104 (2012).

[9] S. Kristyán and P. Pulay, Chem. Phys. Lett. 229, 175 (1994).

[10] E. Hult, Y. Andersson, B. I. Lundqvist, and D. C. Langreth, Phys. Rev. Lett. 77, 2029 (1996).

[11] W. Kohn, Y. Meir, and D. E. Makarov, Phys. Rev. Lett. 80, 4153 (1998).
[12] H. Rydberg, M. Dion, N. Jacobson, E. Schröder, P. Hyldgaard, S. I. Simak, D. C. Langreth, and B. I. Lundqvist, Phys. Rev. Lett. 91, 126402 (2003).

[13] C. T. Campbell, Surf. Sci. 157, 43 (1985).

[14] D. Schmeisser and K. Jacobi, Surf. Sci. 156, 911 (1985).

[15] M. Spruit, E. Kuipers, F. Geuzebroek, and A. Kleyn, Surf. Sci. 215, 421 (1989).

[16] F. Besenbacher and J. K. Nørskov, Prog. Surf. Sci. 44, 5 (1993).

[17] S. Lacombe, F. Cemi, P. He, H. Dietrich, P. Geng, M. Rocca, and K. Jacobi, Surf. Sci. 368, 38 (1996).

[18] I. Goikoetxea, J. Beltrán, J. Meyer, J. I. Juaristi, M. Alducin, and K. Reuter, New J. Phys. 14, 013050 (2012).

[19] J. P. Perdew, K. Burke, and M. Ernzerhof, Phys. Rev. Lett. 77, 3865 (1996).

[20] S. Lorenz, A. Groß, and M. Scheffler, Chem. Phys. Lett. 395, 210 (2004).

[21] S. Lorenz, M. Scheffler, and A. Groß, Phys. Rev. B 73, 115431 (2006).

[22] J. Behler, S. Lorenz, and K. Reuter, J. Chem. Phys. 127, 014705 (2007).

[23] A. Raukema, R. J. Dirksen, and A. W. Kleyn, J. Chem. Phys. 103, 6217 (1995).

[24] J. Gadzuk and S. Holloway, Chem. Phys. Lett. 114, 314 (1985).

[25] P. Riviére, A. Salin, and F. Martín, J. Chem. Phys. 124, 084706 (2006).

[26] I. Goikoetxea, M. Alducin, R. Díez Muiño, and J. I. Juaristi, Phys. Chem. Chem. Phys. 14, 7471 (2012).

[27] S. A. Adelman, J. Chem. Phys. 71, 4471 (1979).

[28] J. C. Tully, J. Chem. Phys. 73, 1975 (1980).

[29] H. F. Busnengo, M. A. Di Césare, W. Dong, and A. Salin, Phys. Rev. B 72, 125411 (2005).

[30] L. Martin-Gondre, M. Alducin, G. A. Bocan, R. Díez Muiño, and J. I. Juaristi, Phys. Rev. Lett. 108, 096101 (2012). 